\title{
INFLUÊNCIA DA REGULAMENTAÇÃO CONTÁBIL DE INSTRUMENTOS FINANCEIROS NA PRODUÇÃO CIENTÍFICA: UMA ANÁLISE BIBLIOMÉTRICA EM PERIÓDICOS NACIONAIS
}

\author{
Influence of Regulation of Accounting of Financial Instruments in \\ Scientific Production: a Bibliometric Analysis in National Journals \\ Influencia de Reglamento de Contabilidad Acerca de Instrumentos \\ Financieros en la Producción Científica: un análisis bibliométrica en \\ revistas nacionales
}

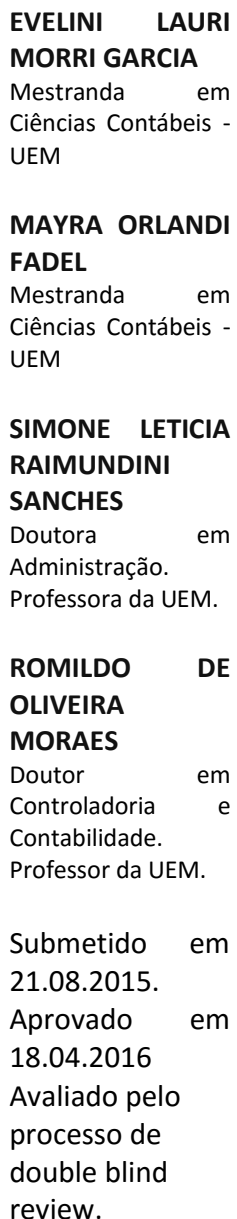

review.

\section{RESUMO}

O objetivo deste trabalho consistiu em verificar a influência das alterações normativas sobre as pesquisas na área contábil de 1995 à 2014 relacionadas aos instrumentos financeiros. Para isso, foi realizado um levantamento bibliográfico das publicações que ocorreram entre 1995 e 2014 com os assuntos "instrumentos financeiros", "hedge" e "derivativos" na base de dados do Portal de Periódicos da CAPES e nos periódicos da área contábil listados no site da Associação Nacional de Programas de Pós-Graduação em Ciências Contábeis (ANPCONT). Foram identificados 43 artigos, os quais compuseram a amostra. Foi verificado que Rodrigo Fernandes Malaquias, Roberto Carlos Klann e Jorge Ribeiro Toledo Filho possuem a maior produção científica sobre o tema, com três artigos cada. As publicações estão concentradas em periódicos de classificação Qualis-CAPES A2 e B1 e o enfoque mais abordado foi a evidenciação, correspondendo a $32,56 \%$ dos estudos analisados. Com relação às alterações das normas contábeis, constatou-se influência significativa das mudanças na regulamentação ocorridas de 2007 à 2010, que motivou $39,53 \%$ dos trabalhos analisados, demonstrando que a convergência aos padrões internacionais de contabilidade representou um nicho de estudos. A IAS 32 e a IAS 39 foram os regulamentos mais citados, presente em $15,29 \%$ das publicações, indicando a influência das normas internacionais em pesquisas nacionais.

Palavras-chave: regulamentação; instrumentos financeiros; pesquisas.

\section{ABSTRACT}

The objective of this study was to verify the influence of regulatory changes on the accounting research area 1995 to 2014 related to financial instruments. For this, a literature review of publications that occurred between 1995 and 2014 with the subjects "financial instruments" was held, "hedge" and "derivatives" in the CAPES Journal Portal database and the journals of accounting area listed on the website the National Association of Graduate Programs in Accounting (ANPCONT). 43 articles, which composed the sample were identified. It was found that Rodrigo Fernandes Malachi, Roberto Carlos and Jorge Klann RibeiroToledo Son have the highest scientific literature on the subject, with three items each. The publications are concentrated in classification Qualis-Capes A2 and B1 and most discussed approach was the disclosure, corresponding to $32.56 \%$ of the analyzed studies. With regard to changes in accounting standards, there was significantly influenced by changes in regulation occurred 2007 to 2010 that motivated $39.53 \%$ of the analyzed studies, demonstrating that convergence with international accounting standards represented a niche studies. IAS 32 and IAS 39 were the most cited regulations, present in $15.29 \%$ of the publications, indicating the influence of international standards in national surveys.

Keywords: regulation; financial instruments; research. 


\section{RESUMEN}

El objetivo de este estudio fue verificar la influencia de los cambios regulatorios en el área de la investigación contable 1995-2014 relacionados con los instrumentos financieros. Para ello, una revisión bibliográfica de las publicaciones que se produjeron entre 1995 y 2014 con los temas "instrumentos financieros" se llevó a cabo, "cobertura" y "derivados" en la base de datos CAPES Diario Portal y las revistas del área contable que aparece en el sitio web la Asociación Nacional de Programas de Postgrado en Contabilidad (ANPCONT). Se identificaron 43 artículos que componen la muestra. Se encontró que Rodrigo Fernandes Malaquías, Roberto Carlos Klann e Jorge Ribeiro Toledo Filho tienen la más alta literatura científica sobre el tema, con tres puntos cada uno. Las publicaciones se concentran en la clasificación Qualis-Capes A2 y B1 y el enfoque más discutido fue la revelación, que corresponde a $32,56 \%$ de los estudios analizados. Con respecto a los cambios en las normas contables, no fue influenciada significativamente por los cambios en la regulación ocurrieron desde 2007 hasta 2010 que motivó 39,53\% de los estudios analizados, lo que demuestra que la convergencia con las normas internacionales de contabilidad representados unos estudios especializados. IAS 32 y IAS 39 fueron los más citados reglamentos, presentes en $15,29 \%$ de las publicaciones, lo que indica la influencia de las normas internacionales en las encuestas nacionales.

Palabras clave: regulación; instrumentos financieros; investigación. 


\section{INTRODUÇÃO}

A produção científica em contabilidade acompanha o desenvolvimento da profissão contábil, e, portanto, vivencia nos últimos anos uma corrente de novidades, em especial na área financeira. A convergência aos padrões internacionais de contabilidade influencia as pesquisas contábeis, dirimindo dúvidas sobre as regras de contabilidade e colaborando com o atendimento do objetivo da Ciência Contábil.

Visto que a produção científica investiga os mais variados aspectos da profissão contábil, é importante que sejam feitas avaliações sobre os estudos efetuados sobre as práticas de contabilidade e suas implicações no dia-a-dia das organizações. Dentre os estudos bibliométricos que pesquisaram a produção científica na área de contabilidade financeira e que foram publicados em periódicos brasileiros da área contábil, encontram-se Machado e Garcia (2014) que investigaram o risco cambial oriundo do uso de derivativos enquanto tema de pesquisas. Ou seja, apenas uma pesquisa bibliométrica relatou aspectos sobre instrumentos financeiros, mesmo este sendo um dos atuais desafios para a normatização contábil. A preocupação sobre a amplitude das pesquisas sobre este tema deve-se ao fato de que os órgãos regulamentadores empregaram diversas melhorias na normatização dos instrumentos financeiros devido a complexidade no registro de transações deste tipo e na ausência de informações fidedignas aos usuários externos.

Considerando que em 1995 ocorreu a emissão da primeira norma contábil brasileira sobre instrumentos financeiros, o presente estudo tem como questão norteadora: qual o estado da arte na produção científica sobre instrumentos financeiros no contexto contábil brasileiro de 1995 até 2014 em relação às normas contábeis? Sendo assim, esta pesquisa tem por objetivo verificar a influência das alterações normativas sobre as pesquisas na área contábil de 1995 à 2014 relacionadas aos instrumentos financeiros.

Este estudo se justifica pela complexidade no uso de instrumentos financeiros, constituindo um tema controverso para a contabilidade e demandando discussões aprofundadas para alcançar o seu desenvolvimento (CHATHAM; LARSON; VIETZE, 2010). A pesquisa sobre os estudos a respeito de instrumentos financeiros colabora com as investigações acadêmicas em variados aspectos, visto que ressaltam os reflexos da convergência das normas internacionais de contabilidade, das práticas de contabilidade condicionadas à normatização, além de elencar oportunidades de novos estudos. Estas características também apoiam o desenvolvimento da profissão contábil, pois, debatem sobre o tema por intermédio da indicação dos estudos que ajudam a compreender com maior facilidade os aspectos complexos que comprometem o entendimento, e, consequentemente, a aplicação das normas.

\section{REFERENCIAL TEÓRICO}

O referencial teórico aborda o desenvolvimento dos instrumentos financeiros, e sua respectiva regulação contábil, em âmbito internacional e nacional.

\subsection{O Desenvolvimento dos Instrumentos Financeiros e a Regulação Contábil Internacional}

Os instrumentos financeiros foram utilizados, inicialmente, como facilitadores de transferência de recursos entre captadores e credores (LOPES; GALDI; LIMA, 2011). Contudo, passaram por modificações devido a necessidade de proteger as empresas perante a 
volatilidade instaurada no mercado, sendo utilizados como ferramenta de gerenciamento de risco de mercado (YOUNG, 1996). Mesmo havendo um risco intrínseco na utilização de instrumentos financeiros, devido a possibilidade de variação do seu valor, ainda assim houve aceitação massiva destes instrumentos (YOUNG, 1996; CHATHAM, LARSON, VIETZE, 2010).

Em março de 1986, surgiu a primeira norma contábil que delineou o tratamento que deveria ser aplicado aos instrumentos financeiros. O International Accounting Standards Committee (IASC), atual International Accounting Standards Board (IASB), emitiu a IAS 25 que apresentou os princípios gerais sobre a contabilização da maioria dos instrumentos financeiros. Após críticas dos stakeholders, o IASC observou que questões menos controversas de divulgação e apresentação poderiam ser abordados de forma satisfatória, originando a IAS 32 (Financial Instruments: Presentation) em 1995. Em junho de 1998, o FASB emitiu o SFAS 133 que teve como objetivo orientar a contabilidade de derivativos e hedges com a adoção do valor justo.

Discussões mais aprofundadas sobre a questão do reconhecimento e mensuração dos instrumentos financeiros continuaram, de modo que, em 1998, foi emitida a IAS 39 Financial Instruments: Recognition and Measurement. Esta norma propôs a revisão da IAS 32 e determinou que a avaliação pelo valor justo fosse utilizada para quase todos os instrumentos financeiros, semelhante ao SFAS no 133 (CHATHAM; LARSON; VIETZE, 2010). Em setembro de 2000 ocorreu a primeira revisão da IAS 39, mesmo ano da emissão da SFAS 140 que delineou o tratamento das transferências de instrumentos financeiros e da extinção de passivos financeiros.

A parceira feita entre o IASB e IASC, em 2002, resultou na emissão da SFAS 150 e na alteração das IAS 32 e 39, em 2003 (DELOITTE, 2014; FASB, 2014). No ano seguinte houve nova ementa à IAS 39 que indicou o reconhecimento inicial dos ganhos e perdas com instrumentos financeiros.

Em 2005 houve a emissão da IFRS 7 - Financial Instruments: Disclosures substituindo a IAS 32 e abordando a divulgação dos instrumentos financeiros (IFRS, 2014). A IAS 39 foi alterada, definindo o valor justo para a mensuração de todos os instrumentos financeiros. Em 2007 foi emitida a SFAS 159 que determinou o uso do valor justo em ativos e passivos financeiros. No ano seguinte, em 2008, a IAS 39 foi substituída pela IFRS 9 - Financial Instruments. Em 2009 também ocorreu a emissão da SFAS 161 que promoveu alterações na SFAS 133 sobre derivativos e hedges e a emissão da SFAS 166 que dispôs sobre transferências de ativos financeiros.

Em relação à IFRS 7 foram efetuadas diversas melhorias. A última delas, em 2013, adicionou novas regras de divulgação, e, em 2014, incluiu a gestão de contratos e as demonstrações financeiras intermediárias consolidadas (DELOITTE, 2014). Sobre a IFRS 9, também houve uma série de melhorias, sendo que em 2014 foi emitida uma versão substituindo todas as versões anteriores e finalizando a última fase da complementação das normas sobre instrumentos financeiros (IFRS, 2014).

\subsection{Normatização Contábil Brasileira dos Instrumentos Financeiros}

No contexto nacional, a regulamentação dos novos instrumentos financeiros só teve início em 1994, pelo Conselho Monetário Nacional (CMN), com a emissão da Resolução 2.042/94 autorizando as instituições financeiras e similares a realizarem operações de swap, sendo posteriormente, revisadas pelas Resoluções CMN 2.138/94 e 2.149/95 (NAKAMURA, 2003). Como até 1995 as operações com derivativos possuíam tratamento off-balance sheet, 
a partir da emissão da Instrução CVM no 235/95, a divulgação dessas operações passou a ser obrigatória para todas as empresas (CVM, 1995; NAKAMURA, 2003; DARÓS, BORBA, 2005).

As circulares do Banco Central (BACEN) no 3.068/01, no 3.082/02, n 3.086/02 e no 3.082/02 foram desenvolvidas com base nas determinações das normas internacionais (IAS 32 e IAS 39), contudo, adaptadas para a realidade das instituições financeiras. Assim, para as instituições não financeiras, a atualização das normas sobre instrumentos financeiros ocorreu apenas por meio da harmonização contábil ocorrida no Brasil com o advento da Lei no 11.638/07.

Inicialmente, foi emitida a Deliberação da Comissão de Valores Mobiliários (CVM) no 550, em 2008, estabelecendo critérios quantitativos na evidenciação de instrumentos financeiros. Ainda em 2008, a Deliberação CVM 550/08 foi convertida na Instrução CVM no 475/08, revogando a Instrução CVM no 235/95 e aprovando o CPC 14 - Instrumentos Financeiros: Reconhecimento, Mensuração e Evidenciação.

Este pronunciamento trouxe alterações nos fundamentos dos registros contábeis para todas as organizações, uma vez que incorporou o conceito de mensuração pelo valor justo, até então aplicado apenas em instituições reguladas pelo Banco Central do Brasil e SUSEP (FIPECAFI; ERNST \& YOUNG, 2008).

Porém, em virtude do CPC 14 ser implementado na primeira fase de convergência das normas internacionais, seu objetivo foi condensar as instruções contidas nos regulamentos internacionais e orientar quanto aos instrumentos financeiros mais corriqueiros. As alterações observadas nas normas internacionais (IAS 32, IAS 39, IFRS 7) foram traduzidas e adaptadas na segunda fase da convergência das normas, em 2009, quando foi emitido o CPC 38 (Instrumentos Financeiros: Reconhecimento e Mensuração) que corresponde à IAS 39, o CPC 39 (Instrumentos Financeiros: Apresentação) baseado na IAS 32 e o CPC 40 (Instrumentos Financeiros: Evidenciação) correspondente ao IFRS 7. O CPC 14 foi convertido na Orientação OCPC 03 refletindo a complexidade da normatização do assunto, que passou a ter maior detalhamento de informações.

Em 2012 o CPC 40 foi revisado devido as alterações ocorridas nas normas internacionais (CPC, 2012; DELOITTE, 2014). Com as recentes alterações na IFRS 7 e na IFRS 9, espera-se que os pronunciamentos técnicos brasileiros correspondentes a estas normas internacionais sejam revisados a fim de absorver a consolidação que as normas sobre instrumentos financeiros galgaram no âmbito internacional.

\section{METODOLOGIA}

A pesquisa é caracterizada como descritiva quanto ao seu objetivo e como qualitativa quanto a abordagem do problema. Em relação aos procedimentos de coleta de dados foi adotada a pesquisa bibliográfica, onde foi utilizada a base de dados de periódicos da CAPES e os periódicos brasileiros da área contábil listados no site da Associação Nacional de Programas de Pós-Graduação em Ciências Contábeis (ANPCONT), sendo encontradas 43 pesquisas que compõem a amostra do presente estudo. A busca foi realizada considerando todos os artigos que foram publicados em periódicos brasileiros da área contábil. Foram coletados os artigos que possuíam os termos "instrumentos financeiros", ou "derivativos" ou "hedge" como assunto. Foi definido o período de 1995 a 2014 como parâmetro da busca, visto que as alterações mais significativas na normatização contábil ocorreram nesse período, conforme destacado no capítulo da revisão da literatura. 
A análise bibliométrica foi empregada como técnica qualitativa para ponderar os dados, conjuntamente à técnica de análise de conteúdo. A categorização utilizada para a tabulação dos dados incorporou os seguintes itens: (i) ano de publicação; (ii) identificação e quantidade dos autores; (iii) vínculo institucional dos autores; (iv) título; (v) periódico; (vi) classificação do periódico; (vii) citações; (viii) foco da pesquisa; (ix) teoria de base; (x) período analisado; e, (xi) norma contábil utilizada. Os itens foram extraídos dos artigos, quando identificáveis.

Para tabulação do item (iii) foram considerados os vínculos institucionais de todos os autores, considerando a primeira identificação. O item vi considera a classificação Qualis da Capes (entre $\mathrm{A} 1$ e C). $\mathrm{O}$ item vii identifica as quantidades de citações obtidas em cada artigo por intermédio da ferramenta Google Scholar. $O$ item xi considerada as normas por período de emissão, demonstrado no Quadro 1.

Quadro 1 - Marcos das mudanças nas normas contábeis

\begin{tabular}{|c|c|c|}
\hline Período & Normas internacionais & Normas brasileiras \\
\hline $1995-1998$ & $\begin{array}{c}\text { Emissão da IAS } 32 \text { (1995); emissão da IAS } 39 \text { (1998); } \\
\text { revisão da IAS } 32 \text { pela IAS } 39 \text { (1998); emissão da SFAS } \\
133 \text { (1998) }\end{array}$ & Instrução CVM 235/95 \\
\hline $1999-2002$ & Revisão da IAS 39 (2000); emissão da SFAS 140 (2000) & $\begin{array}{l}\text { Circular BACEN n } 3.068 / 01 \\
\text { Circular BACEN no 3.082/02; } \\
\text { Circular BACEN n } 3.086 / 02 \\
\text { Circular BACEN nㅇ 3.150/02; }\end{array}$ \\
\hline $2003-2006$ & $\begin{array}{c}\text { Emissão da SFAS } 150 \text { (2003); revisão da IAS } 32 \text { (2003); } \\
\text { ementa à IAS } 39 \text { (2003); ementa à IAS } 39 \text { (2004); } \\
\text { alteração da IAS } 39 \text { (2005); substituição da IAS } 32 \text { pelo } \\
\text { IFRS } 7 \text { (2005) }\end{array}$ & \\
\hline $2007-2010$ & $\begin{array}{l}\text { SFAS } 159 \text { (2007); alteração da IFRS } 7 \text { (2008); alterações } \\
\text { na IAS } 39 \text { (2008); SFAS } 161 \text { (2008); Substituição da IAS } \\
39 \text { pelo IFRS } 9 \text { (2009); SFAS } 166 \text { (2009); alterações na } \\
\text { IFRS } 7 \text { (2010); reedição da IFRS } 9 \text { (2010) }\end{array}$ & $\begin{array}{c}\text { Promulgação da Lei 11.638/07; } \\
\text { Deliberação CVM 550/08; Instrução } \\
\text { CVM 475/08, CPC 14 (2008); OCPC } \\
03 \text { (2009); CPC } 38 \text { (2009), CPC } 39 \\
\text { (2009), CPC } 40 \text { (2009) }\end{array}$ \\
\hline $2011-2014$ & $\begin{array}{l}\text { Alterações na IFRS } 9 \text { (2011); restrições das alterações } \\
\text { do IFRS } 9 \text { em } 2010 \text { (2012); alterações na IFRS } 7 \text { (2013); } \\
\text { alterações na IFRS } 9 \text { (2013); alterações na IFRS } 7 \\
\text { (2014); consolidação das alterações da IFRS } 9 \text { (2014) }\end{array}$ & Revisão do CPC 40 (2012) \\
\hline
\end{tabular}

FONTE: DADOS DA PESQUISA

A normatização contábil utilizada estende-se em um período de 20 anos (1995 à 2014) e foi classifica a fim de agregar períodos com o mesmo espaço de tempo. Considerando os padrões de categorização definidos nesta pesquisa, após a leitura dos artigos na íntegra, utilizou-se o Microsoft Excel ${ }^{\circledR}$ para tabulação dos dados.

\section{ANÁLISE DOS DADOS}

A partir da análise da produção científica brasileira de contabilidade nos últimos 20 anos, acerca dos instrumentos financeiros, identificou-se que estes estudos contemplam diversas abordagens, conforme demonstrado no Quadro 2. 
Tabela 1 - Abordagem das pesquisas sobre instrumentos financeiros

\begin{tabular}{|c|c|c|c|}
\hline Enfoque das pesquisas & Autores & $\begin{array}{c}\text { Qtde. de } \\
\text { publicações }\end{array}$ & Freq. \\
\hline Evidenciação & $\begin{array}{l}\text { Costa Junior (2003), Darós e Borba (2005), Barros e } \\
\text { Lopes (2006), Murcia e Santos (2009), Santos et al. } \\
\text { (2010), Quirino, Boente e Melo (2011), Peixoto e } \\
\text { Malaquias (2012), Santos et al. (2012), Alves e Graça } \\
\text { (2013), Malaquias e Lemes (2013), Santos et al. (2013), } \\
\text { Silva, Machado e Hein (2013), Ambrozini (2014) e } \\
\text { Bianchi et al. (2014). }\end{array}$ & 14 & $32,56 \%$ \\
\hline Hedge accounting & $\begin{array}{l}\text { Amaral, Souza e França (2000), Lopes e Santos (2003), } \\
\text { Kimura e Perera (2005), Lameira, Figueiredo e Ness } \\
\text { (2005), Hoji e Costa (2006), Zen, Yatabe e Carvalho } \\
\text { (2006), Galdi e Guerra (2009), Souza et al. (2011), Matos } \\
\text { et al. (2013), Sticca e Nakao (2013) e Vieira et al. (2014). }\end{array}$ & 11 & $25,58 \%$ \\
\hline Uso dos derivativos & $\begin{array}{l}\text { Lima e Beuren (2000), Carneiro e Sherris (2008), } \\
\text { Gimenes (2008), Costa e Piacenti (2008), Burlá e } \\
\text { Gonçalves (2010), Klann, Cunha e Toledo Filho (2010), } \\
\text { Machado e Garcia (2014), Marques e Petri (2014) e } \\
\text { Portulhak et al. (2014). }\end{array}$ & 9 & $20,93 \%$ \\
\hline $\begin{array}{l}\text { Comparativo entre } \\
\text { normas }\end{array}$ & $\begin{array}{l}\text { Moreira e Lima (2003), Morch et al. (2009) e Sayed et al. } \\
\text { (2013) }\end{array}$ & 3 & $6,98 \%$ \\
\hline $\begin{array}{l}\text { Evolução da } \\
\text { regulamentação }\end{array}$ & Lopes e Lima (2001) e Amaral (2003) & 2 & $4,65 \%$ \\
\hline $\begin{array}{c}\text { Contabilização dos } \\
\text { derivativos }\end{array}$ & Cova (2006) & 1 & $2,33 \%$ \\
\hline $\begin{array}{l}\text { Convergência contábil } \\
\text { das normas }\end{array}$ & Strouhal (2009) & 1 & $2,33 \%$ \\
\hline $\begin{array}{l}\text { Determinantes da } \\
\text { evidenciação }\end{array}$ & Mapurunga et al. (2011) & 1 & $2,33 \%$ \\
\hline $\begin{array}{l}\text { Percepção da } \\
\text { regulamentação }\end{array}$ & Sá e Malaquias (2012) & 1 & $2,33 \%$ \\
\hline \multicolumn{2}{|r|}{ Total } & 43 & $100,00 \%$ \\
\hline
\end{tabular}

FONTE: DADOS DA PESQUISA

Observa-se que o tema instrumentos financeiros é mais estudado quanto à perspectiva da evidenciação, com 14 pesquisas (32,56\%). Ainda destacam-se as pesquisas sobre hedge accounting (11 artigos) e uso dos derivativos (9 artigos). Desta forma, pode-se verificar que as pesquisas priorizaram investigar a informação que os investidores recebem a respeito dos instrumentos financeiros e aprofundar conhecimento sobre o uso dos instrumentos financeiros secundários. Além disso, busca-se verificar a transparência quanto ao uso de instrumentos financeiros.

A Tabela 2 demonstra a distribuição da produção científica no período de 2000 a 2014, segregada por número de autores e ano de publicação.

Tabela 2 - Produção científica por período e quantidade de autores

\begin{tabular}{|c|c|c|c|c|c|c|c|}
\hline \multirow[t]{2}{*}{ Ano } & \multicolumn{5}{|c|}{ Quantidade de Autores } & \multirow{2}{*}{ Total } & \multirow{2}{*}{ Frequência } \\
\hline & 1 autor & 2 autores & 3 autores & 4 autores & 5 autores & & \\
\hline 2000 & 0 & 1 & 1 & 0 & 0 & 2 & 4,65 \\
\hline 2001 & 0 & 1 & 0 & 0 & 0 & 1 & $2,33 \%$ \\
\hline 2002 & 0 & 0 & 0 & 0 & 0 & 0 & $0,00 \%$ \\
\hline 2003 & 2 & 2 & 0 & 0 & 0 & 4 & $9,30 \%$ \\
\hline
\end{tabular}




\begin{tabular}{|c|c|c|c|c|c|c|c|}
\hline 2004 & 0 & 0 & 0 & 0 & 0 & 0 & $0,00 \%$ \\
\hline 2005 & 0 & 2 & 1 & 0 & 0 & 3 & $6,98 \%$ \\
\hline 2006 & 1 & 2 & 1 & 0 & 0 & 4 & $9,30 \%$ \\
\hline 2007 & 0 & 0 & 0 & 0 & 0 & 0 & $0,00 \%$ \\
\hline 2008 & 1 & 2 & 0 & 0 & 0 & 3 & $6,98 \%$ \\
\hline 2009 & 1 & 2 & 0 & 0 & 1 & 4 & $9,30 \%$ \\
\hline 2010 & 0 & 1 & 1 & 0 & 1 & 3 & $6,98 \%$ \\
\hline 2011 & 0 & 0 & 1 & 2 & 0 & 3 & $6,98 \%$ \\
\hline 2012 & 0 & 2 & 0 & 1 & 0 & 3 & $6,98 \%$ \\
\hline 2013 & 0 & 3 & 1 & 2 & 1 & 7 & $16,28 \%$ \\
\hline 2014 & 1 & 2 & 0 & 3 & 0 & 6 & $13,95 \%$ \\
\hline Total & 6 & 20 & 6 & 8 & 3 & 43 & \\
\hline$\%$ & $13,95 \%$ & $46,51 \%$ & $13,95 \%$ & $18,60 \%$ & $6,98 \%$ & $100 \%$ & $100 \%$ \\
\hline
\end{tabular}

Fonte: Dados da pesquisa

Durante o período de 1995 à 1999 não foi localizada nenhuma publicação sobre instrumentos financeiros em revistas brasileiras da área contábil. As publicações encontradas ficaram concentradas, em sua maioria, nos anos de 2003 (9,3\%), 2006 (9,3\%), 2009 (9,3\%), $2013(16,28 \%$ ) e 2014 (13,95\%) representando 58,13\% do total da produção acadêmica identificada. É possível verificar que a produção científica, acerca do tema instrumentos financeiros, obteve seu ápice em 2013 e 2014, indicando que houve maior quantidade de estudos sobre este assunto após ocorrer, no Brasil, a convergência aos padrões internacionais de contabilidade. Foi predominante a parceria entre 2 autores com $46,51 \%$ dos resultados (20 artigos).

A Tabela 3 demonstra o vínculo institucional dos autores. Observa-se que os estudos sobre instrumentos financeiros na área contábil encontram-se distribuídos entre pesquisadores de 33 instituições de ensino, sendo 29 universidades brasileiras e 4 universidades estrangeiras (Portugal, Estados Unidos, Austrália, República Theca), corroborando a relevância observada sobre o tema. O predomínio de publicações está vinculada à Universidade de São Paulo $(21,65 \%)$ e pode ser explicado pelo fato de ser o principal polo de pesquisa em contabilidade no Brasil.

Tabela 3 - Vínculo institucional dos autores

\begin{tabular}{lcc}
\hline Instituição de Ensino Superior & Qtde. & Frequência \\
\hline USP - Universidade de São Paulo (SP) & 21 & $21,65 \%$ \\
FURB - Universidade Regional de Blumenau (SC) & $5,15 \%$ \\
UFSC - Universidade Federal de Santa Catarina (SC) & $5,15 \%$ \\
UnB - Universidade de Brasília (DF) & 5 & $5,15 \%$ \\
FECAP - Fundação Escola de Comércio Álvares Penteado (SP) & $4,12 \%$ \\
PUC-SP - Pontifícia Universidade Católica (SP) & 4 & $4,12 \%$ \\
UFC - Universidade Federal do Ceará (CE) & 4 \\
UFU - Universidade Federal de Uberlândia (MG) & 4 & $4,12 \%$ \\
FGV - Fundação Getúlio Vargas (SP) & 3 & $3,09 \%$ \\
PUC-Rio - Pontifícia Universidade Católica (RJ) & 3 & $3,09 \%$ \\
UERJ - Universidade Estadual do Rio de Janeiro (RJ) & 3 \\
UFMG - Universidade Federal de Minas Gerais (MG) & 3 \\
UFPR - Universidade Federal do Paraná (PR) & 3 & $3,09 \%$ \\
UFRGS - Universidade Federal do Rio Grande do Sul (RS) & $3,09 \%$ \\
UFRN - Universidade Federal do Rio Grande do Norte (RN) & $3,09 \%$ \\
ESCE - Escola Superior de Ciências Empresariais do Instituto Politécnico de Setúbal & 2 & $3,09 \%$ \\
\hline \hline
\end{tabular}




\begin{tabular}{llc}
\hline (Portugal) & & \\
FUCAPE - Business School (ES) & 2 & $2,06 \%$ \\
FUMEC - Universidade Fundação Mineira de Educação e Cultura (MG) & 2 & $2,06 \%$ \\
Mackenzie - Universidade Presbiteriana Mackenzie (SP) & 2 & $2,06 \%$ \\
UFPE - Universidade Federal do Pernambuco (PE) & 2 & $2,06 \%$ \\
UFV - Universidade Federal de Viçosa (MG) & 2 & $2,06 \%$ \\
Ohio University (EUA) & 1 & $1,03 \%$ \\
\hline PUC-MG - Pontifícia Universidade Católica (MG) & 1 & $1,03 \%$ \\
School of Actuarial Studies University of New South Wales (Austrália) & 1 & $1,03 \%$ \\
UDESC - Universidade do Estado de Santa Catarina (SC) & 1 & $1,03 \%$ \\
UEM - Universidade Estadual de Maringá (PR) & 1 & $1,03 \%$ \\
UFAM - Universidade Federal do Amazonas (AM) & 1 & $1,03 \%$ \\
UFBA - Universidade Federal da Bahia (BA) & 1 & $1,03 \%$ \\
UFF - Universidade Federal Fluminense (RJ) & 1 & $1,03 \%$ \\
UFPB - Universidade Federal da Paraíba (PB) & 1 & $1,03 \%$ \\
UNIPAR - Universidade Paranaense (PR) & 1 & $1,03 \%$ \\
Universidade de Economía Praga (República Theca) & 1 & $1,03 \%$ \\
UNOCHAPECÓ - Universidade Comunitária Regional de Chapecó (SC) & 1 & $1,03 \%$ \\
Total & 97 & $\mathbf{1 0 0 , 0 0 \%}$ \\
\hline
\end{tabular}

Fonte: Dados da pesquisa

No quesito regionalidade, dentre as instituições que os autores estão vinculados, 34 artigos estão vinculados à instituições que estão localizadas no Estado de São Paulo, seguido pelo Estado de Minas Gerais e de Santa Catarina, ambos com 12 e Rio de Janeiro com 7. Isso mostra que as instituições de ensino da região Sudeste concentra a produção científica sobre o tema.

A Tabela 4 demonstra os periódicos onde foram publicados os artigos sobre instrumentos financeiros. Salienta que esta pesquisa limitou-se ao levantamento de publicações na área contábil.

Tabela 4 - Produção científica por periódico

\begin{tabular}{|c|c|c|c|}
\hline Periódico & $\begin{array}{l}\text { Qualis- } \\
\text { Capes }\end{array}$ & $\begin{array}{c}\text { № de } \\
\text { Publicações }\end{array}$ & $\begin{array}{c}\text { \% de } \\
\text { Frequência }\end{array}$ \\
\hline Revista Contabilidade \& Finanças & $\mathrm{A} 2$ & 11 & \multirow{2}{*}{$27,91 \%$} \\
\hline BBR - Brazilian Business Review & A2 & 1 & \\
\hline RCO - Revista de Contabilidade e Organizações & B1 & 4 & \multirow{4}{*}{$27,91 \%$} \\
\hline Revista Contemporânea de Contabilidade & B1 & 4 & \\
\hline Contabilidade Vista e Revista & B1 & 2 & \\
\hline Revista Universo Contábil & B1 & 2 & \\
\hline Revista Ambiente Contábil & B2 & 3 & \multirow{6}{*}{$23,25 \%$} \\
\hline Contabilidade, Gestão e Governança & B2 & 2 & \\
\hline Sociedade, Contabilidade e Gestão & B2 & 2 & \\
\hline Enfoque Reflexão Contábil & B2 & 1 & \\
\hline RePec - Revista de Educação e Pesquisa em Contabilidade & B2 & 1 & \\
\hline Revista de Administração, Contabilidade e Economia & B2 & 1 & \\
\hline Revista de Contabilidade do Mestrado de Ciências Contábeis da UERJ & B3 & 3 & \multirow{7}{*}{$20,93 \%$} \\
\hline ConTexto & B3 & 1 & \\
\hline Revista Contabilidade e Controladoria & B3 & 1 & \\
\hline Revista de Contabilidade do Mestrado de Ciências Contábeis da UFRJ & B3 & 1 & \\
\hline Revista de Gestão, Finanças e Contabilidade & B3 & 1 & \\
\hline Revista de Informação Contábil & B3 & 1 & \\
\hline \multirow[t]{2}{*}{ Revista Pensar Contábil } & B3 & 1 & \\
\hline & Total & 43 & $100 \%$ \\
\hline
\end{tabular}

Fonte: Dados da pesquisa 
Foram identificados 19 periódicos com publicações sobre o tema instrumentos financeiros. A Revista Contabilidade \& Finanças, que possui a maior quantidade de publicações (11 artigos), está vinculado ao Programa de Pós-Graduação da Universidade de São Paulo, cuja instituição concentra $21,65 \%$ dos autores dos artigos analisados (Tabela 3 ). Ao verificar os 11 artigos publicados nesta Revista percebe-se que existem 4 estudos com endogenia, visto que foram realizados por autores vinculados à USP. É possível observar que as publicações da Revista Contabilidade \& Finanças ocorreram entre 2001 e 2011. Além disso, verifica-se que até o ano de 2009 existe predomínio de artigos publicados em revistas com Qualis A2 e B1, enquanto que a partir do ano de 2010 houve aumento da quantidade de artigos publicados em revistas com Qualis B2 e B3.

Ao analisar os estudos da amostra é possível identificar que durante o período de 2000 a 2005, período em que o tema instrumentos financeiros era bastante incipiente no Brasil, houve predominância de publicações da Revista Contabilidade \& Finanças, visto que entre os 10 artigos publicados neste espaço tempo, 7 são deste periódico. A partir do ano de 2006 iniciou a pulverização das revistas que publicaram artigos sobre instrumentos financeiros, à medida que o assunto passou a ser mais discutido entre os profissionais da contabilidade.

$\mathrm{Na}$ Tabela 5 estão relacionados os artigos mais citados sobre o tema, conforme o critério do número de citações obtidas na ferramenta Google Scholar. Novamente, observase que as publicações tratadas como relevantes e com maior frequência de citação são aquelas publicadas, predominantemente, nos periódicos de Qualis A2. Também encontra-se um artigo, entre os mais prolíficos, com classificação Qualis B1.

\section{Tabela 5 - Artigos mais prolíficos}

\begin{tabular}{|c|c|c|c|c|}
\hline Título & Autores & Ano & Citações & Qualis \\
\hline $\begin{array}{l}\text { Evidenciação de instrumentos financeiros derivativos nas } \\
\text { demonstrações contábeis: uma análise das empresas brasileiras }\end{array}$ & DARÓS, L. L.; BORBA, J. A. & 2005 & 24 & A2 \\
\hline $\begin{array}{l}\text { Uma avaliação do nível de evidenciação das companhias } \\
\text { abertas, no Brasil, no tocante aos instrumentos financeiros }\end{array}$ & COSTA JUNIOR, J. V. & 2003 & 21 & A2 \\
\hline $\begin{array}{l}\text { Perspectivas para a pesquisa em contabilidade: o impacto dos } \\
\text { derivativos }\end{array}$ & LOPES, A. B.; LIMA, I. S. & 2001 & 18 & A2 \\
\hline Derivativos: o que são e a evolução quanto ao aspecto contábil & AMARAL, C. A. L. V. & 2003 & 17 & A2 \\
\hline $\begin{array}{l}\text { Modelo de otimização de gestão de risco em empresas não } \\
\text { financeiras }\end{array}$ & $\begin{array}{l}\text { KIMURA, H.; PERERA, L. C. } \\
\text { J. }\end{array}$ & 2005 & 15 & A2 \\
\hline $\begin{array}{l}\text { Determinantes do nível de disclosure de instrumentos } \\
\text { financeiros derivativos em firmas brasileiras }\end{array}$ & $\begin{array}{l}\text { MAPURUNGA, P. V. R.; } \\
\text { PONTE, V. M. R.; COELHO, } \\
\text { A. C. D.; MENESES; A. F. }\end{array}$ & 2011 & 15 & A2 \\
\hline $\begin{array}{l}\text { Regulação contábil e a divulgação de informações de operações } \\
\text { com instrumentos financeiros derivativos: análise do impacto da } \\
\text { CVM no 566/08 e da CVM no } 475 / 08 \text { no disclosure das } \\
\text { companhias abertas no Brasil }\end{array}$ & $\begin{array}{l}\text { MURCIA, F. D.; SANTOS, } \\
\text { A. }\end{array}$ & 2009 & 8 & B1 \\
\hline $\begin{array}{l}\text { A administração do lucro contábil e os critérios para } \\
\text { determinação da eficácia do hedge accounting: utilização da } \\
\text { correlação simples dentro do arcabouço do SFAS no } 133\end{array}$ & $\begin{array}{l}\text { LOPES, A. B.; SANTOS, N. } \\
\text { S. }\end{array}$ & 2003 & 7 & A2 \\
\hline $\begin{array}{l}\text { Corporate interest rate risk management with derivatives in } \\
\text { Australia: empirical results }\end{array}$ & $\begin{array}{l}\text { CARNEIRO, L. A. F.; } \\
\text { SHERRIS, M. }\end{array}$ & 2008 & 7 & A2 \\
\hline $\begin{array}{l}\text { Utilização de contratos futuros agropecuários no perfil médio de } \\
\text { investimentos dos fundos de pensão no Brasil }\end{array}$ & $\begin{array}{l}\text { COSTA, T. M. T.; } \\
\text { PIACENTI, C. A. }\end{array}$ & 2008 & 5 & A2 \\
\hline
\end{tabular}

Fonte: Dados da pesquisa

O artigo com maior número de citações (24) foi o trabalho de Leandro Luis Darós e José Alonso Borba, a segunda pesquisa mais citada (21) foi de Jorge Vieira Costa Junior e em terceiro lugar ficou o trabalho de Alexsandro Broedel Lopes e Iran Siqueira Lima com 18 
citações. Estes três trabalhos estão publicados na Revista Contabilidade \& Finanças e figuram entre os primeiros 5 artigos publicados acerca do tema instrumentos financeiros no Brasil. Entre os 10 artigos mais prolíficos, 9 deles foram publicados pela Revista Contabilidade \& Finanças e um artigo pertence à RCO - Revista de Contabilidade e Organizações.

A tabela 6 identifica os autores que mais tiveram publicações sobre o tema.

Tabela 6 - Autores com maior volume de produção científica

\begin{tabular}{lccc}
\hline Autor & Titulação & Vínculo Institucional Atual & No de Publicações \\
\hline KLANN, R. C. & Doutor & FURB & 3 \\
MALAQUIAS, R. F. & Doutor & UFU & 3 \\
TOLEDO FILHO, J. R. & Doutor & FURB & 3 \\
GUBIANI, C. A. & Mestre & UNOCHAPECÓ & 2 \\
LOPES, A. B. & Doutor & USP & 2 \\
SANTOS, V. & Mestre & UDESC & 2 \\
\hline
\end{tabular}

Fonte: Dados da pesquisa

Pode-se observar que apenas 3 autores produziram 3 pesquisas, cada um, sobre o tema e que três autores desenvolveram 2 estudos cada. Os demais 91 autores que foram identificados publicaram apenas um estudo cada acerca de assuntos relacionados aos instrumentos financeiros. Dentre os 6 autores com maior produção científica sobre o tema, 2 estão atualmente vinculados à FURB - Universidade Regional de Blumenau. Este resultado pode ser relacionado às informações da Tabela 3 que indica esta universidade como a segunda instituição com maior número de autores de estudos sobre instrumentos financeiros, empatada com outras duas universidades.

A Tabela 7 relaciona os anos em que ocorreram as publicações com os períodos das emissões das normas que os respectivos estudos indicam, cuja classificação está definida no Quadro 1.

Tabela 7 - Produção científica relacionada às alterações das normas

\begin{tabular}{|c|c|c|c|c|c|c|}
\hline \multirow{2}{*}{ Ano de Public. } & \multicolumn{5}{|c|}{ Período de mudança das normas contábeis } & \multirow{2}{*}{$\begin{array}{c}\text { Sem } \\
\text { Referência }\end{array}$} \\
\hline & 1995-1998 & 1999-2002 & 2003-2006 & $2007-2010$ & 2011-2014 & \\
\hline 2000 & 1 & & & & & 1 \\
\hline 2001 & 1 & & & & & \\
\hline \multicolumn{7}{|l|}{2002} \\
\hline 2003 & 3 & 1 & & & & \\
\hline \multicolumn{7}{|l|}{2004} \\
\hline 2005 & 1 & & & & & 2 \\
\hline 2006 & 1 & 2 & & & & 1 \\
\hline \multicolumn{7}{|l|}{2007} \\
\hline 2008 & & & 1 & & & 2 \\
\hline 2009 & 1 & & & 3 & & \\
\hline 2010 & & & & 3 & & \\
\hline 2011 & & & & 2 & & 1 \\
\hline 2012 & 1 & & & 2 & & \\
\hline 2013 & & & 3 & 4 & & \\
\hline 2014 & & & 1 & 3 & & 2 \\
\hline Total & 9 & 3 & 5 & 17 & 0 & 9 \\
\hline Frequência & $20,93 \%$ & $6,98 \%$ & $11,63 \%$ & $39,53 \%$ & $0,00 \%$ & $20,93 \%$ \\
\hline \multicolumn{7}{|c|}{ Fonte: Dados da pesquisa } \\
\hline ISSN:2237-3667 & & & REUNIR | Sc & PB | V. 6 & | jan-abr & \begin{tabular}{l|l|l}
16 & p.74-89
\end{tabular} \\
\hline
\end{tabular}


Observa-se que ocorreu maior concentração das publicações relacionadas com as normas emitidas de 2007 à 2010, sendo este período caracterizado por profundas alterações normativas. Esta análise é coerente com os resultados apresentados na Tabela 2, onde os anos posteriores ao período de 2007 à 2010 apresentaram maiores índices de publicação de artigos sobre instrumentos financeiros.

Também fica evidente que houve considerável quantidade de publicações (9 artigos) relacionadas às alterações normativas ocorridas entre 1995 e 1998. Isto enfatiza como as normas de contabilidade influenciaram o desenvolvimento da pesquisa contábil sobre instrumentos financeiros. Este fato é comprovado pela proficuidade dos artigos indicados na Tabela 5, onde as pesquisas pioneiras sobre instrumentos financeiros, são as principais referências de estudos sobre este tema.

As normas utilizadas nas pesquisas estão evidenciadas na Tabela 8. Como diversos artigos utilizaram mais de uma norma contábil, foram consideradas todas aquelas que representam a plataforma teórica da pesquisa.

Tabela 8 - Publicações vinculadas às normas emitidas

\begin{tabular}{|c|c|c|c|c|c|}
\hline \multirow{2}{*}{ Norma } & \multicolumn{2}{|c|}{ Publicações Vinculadas } & \multirow{2}{*}{ Norma } & \multicolumn{2}{|c|}{ Publicações Vinculadas } \\
\hline & Qde. & Freq. & & Qde. & Freq. \\
\hline IAS 32 & 13 & $15,29 \%$ & Circ. BACEN 3.082/02 & 2 & $2,35 \%$ \\
\hline IAS 39 & 13 & $15,29 \%$ & SFAS 150 & 2 & $2,35 \%$ \\
\hline SFAS 133 & 11 & $12,94 \%$ & IFRS 9 & 2 & $2,35 \%$ \\
\hline Instrução CVM 235/95 & 8 & $9,41 \%$ & Deliberação CVM 550/08 & 2 & $2,35 \%$ \\
\hline CPC 40 & 6 & $7,06 \%$ & Circ. BACEN 3.068/01 & 1 & $1,18 \%$ \\
\hline CPC 14 & 5 & $5,88 \%$ & Circ. BACEN 3.086/02 & 1 & $1,18 \%$ \\
\hline CPC 38 & 4 & $4,71 \%$ & SFAS 161 & 1 & $1,18 \%$ \\
\hline СРC 39 & 4 & $4,71 \%$ & SFAS 140 & 0 & $0 \%$ \\
\hline IFRS 7 & 4 & $4,71 \%$ & Circ. BACEN 3.150/02 & 0 & $0 \%$ \\
\hline Instrução CVM 475/08 & 3 & $3,53 \%$ & SFAS 159 & 0 & $0 \%$ \\
\hline \multirow[t]{2}{*}{ Lei 11.638/09 } & 3 & $3,53 \%$ & SFAS 166 & 0 & $0 \%$ \\
\hline & & & Total & 85 & $100,00 \%$ \\
\hline
\end{tabular}

Fonte: Dados da pesquisa

Os resultados demonstram as normas internacionais IAS 32 e IAS 39 (15,29\% cada) como as principais referências das pesquisas brasileiras sobre instrumentos financeiros, indicando influência das normas internacionais em pesquisas nacionais. Esta observação pode ser compreendida pelo fato de que estes regulamentos foram os precursores da IFRS 7 e da IFRS 9, assim como serviram de base para o desenvolvimento das primeiras normativas brasileiras sobre este tema. Também houve considerável indicação da norma norteamericana SFAS 133 (12,94\%).

As normas exclusivamente brasileiras (Instrução CVM 235/95, CPC 40, CPC 14, CPC 38 e CPC 39, respectivamente) figuram entre as mais citadas representando a preocupação dos pesquisadores em investigar as consequências das primeiras normas contábeis, para empresas não financeiras, originárias do processo de conversão aos padrões internacionais de contabilidade. 


\section{CONSIDERAÇÕES FINAIS}

O objetivo deste trabalho consistiu em verificar a influência das alterações normativas sobre as pesquisas na área contábil de 1995 à 2014 relacionadas aos instrumentos financeiros. Foram identificados 43 artigos publicados, constatando-se que as pesquisa sobre instrumentos financeiros no Brasil são influenciadas pela emissão de normas contábeis relativas a este assunto.

Ao analisar os estudos, é possível verificar que há diferentes focos de pesquisa sobre instrumentos financeiros, contudo, ainda existe concentração de pesquisas sobre evidenciação, hedge accounting e uso de derivativos. Outros enfoques possuem poucos estudos, além da amplitude de temas ainda ser restrita. Isso demonstra que as pesquisas sobre instrumentos financeiros podem ser expandidas em relação à delimitação do tema e em profundidade, principalmente, em virtude das recentes alterações no conjunto de normas destas práticas.

A temática instrumentos financeiros sempre foi, e ainda é, considerada um assunto complexo, tanto para os profissionais da contabilidade, quanto aos pesquisadores. Em virtude desta característica, as pesquisas sobre este tema estiveram, inicialmente, concentradas nos centros de estudos com maior desenvolvimento. Desta forma, identificase que os autores vinculados à USP lideram o ranking de pesquisadores sobre este tema. Ainda que nos últimos anos a pesquisa sobre instrumentos financeiros esteja pulverizada entre periódicos e autores vinculados a universidades de todo o Brasil, ainda é possível constatar que a região sudeste congrega a maior proporção de pesquisas sobre o assunto. Portanto, compreende-se que poucas universidades tem se empenhado em realizar aprofundamentos sobre instrumentos científicos.

Apesar de ser tratado como um assunto de difícil compreensão, percebe-se que os instrumentos financeiros estão sendo difundidos entre o meio contábil. Praticamente todos os primeiros trabalhos desenvolvidos sobre este assunto estavam publicados em periódios com Qualis A2. Com o passar dos anos, o assunto também passou a ser encontrado em periódicos com Qualis B1, B2 e B3. Ou seja, os avaliadores dos periódicos com menor classificação no Qualis precisaram de mais tempo para estar preparados para receber pesquisas sobre este tema, reforçando o entendimento de que a disseminação do conhecimento sobre instrumentos financeiros ainda é restrito e que oferece amplas possibilidades de estudos.

A comunidade de pesquisadores têm percebido as oportunidades que este tema oferece, e, assim, o volume de pesquisas sobre este contexto foi elevado, em 2012 e 2013. Contudo, percebe-se que as publicações em periódicos com Qualis A2 diminuíram e que aumentaram em revistas com menores classificações, o que pode-se compreender que as pesquisas atuais sobre instrumentos financeiros tem atingido um menor nível de qualidade ou relevância. Este pode ser um reflexo da falta de preparo dos pesquisados para tratar este assunto, que é delineado por complexos pressupostos. Um ponto que reforça este entendimento é que, de forma geral, os pesquisadores não dão continuidade às pesquisas sobre o tema.

Acerca das alterações normativas, verificou-se que os estudos sobre este tema passaram a ser desenvolvidos no mesmo ritmo das emissões das regulamentações, atingindo seu ápice $(39,53 \%$ das pesquisas) no período de convergência aos padrões internacionais de contabilidade (2007 a 2010). A IAS 32 e a IAS 39 foram os regulamentos mais citados pelas pesquisas, indicando a influência das normas internacionais em pesquisas 
nacionais. A utilidade da informação despertou a necessidade de conhecer os instrumentos financeiros e como estes interferem nos resultados das entidades. Isto reflete que a mudança não ocorreu apenas nas regras práticas de contabilidade, mas também, na percepção da essência dos fatos organizacionais e seus reflexos no patrimônio das empresas.

Considerando os períodos em que as normas foram emitidas, identifica-se que não foram encontradas pesquisas relativas às normas emitidas de 2011 a 2014 . Este pode ser o reflexo do espaço de tempo que existe entre a submissão dos artigos e sua efetiva publicação. Isso demonstra que quando os artigos são publicados, a eficácia de suas conclusões pode ser prejudicada, caso tenha ocorrido modificação das normas que estruturaram a pesquisa.

Assim, pode-se compreender que o presente estudo oferece oportunidades aos pesquisadores contábeis e demonstra que é preciso observar as atuais questões que envolvem os instrumentos financeiros para que novos estudos sejam elaborados com qualidade. Busca-se motivar investigações que colaborem com a verificação das normas quanto à sua adequação às práticas efetuadas pelas organizações, assim como, busquem levantar evidências empíricas em prol do atendimento das normas existentes. Estes resultados são importantes, pois afetam as instituições de ensino superior e seus centros de pesquisas, as organizações por meio das suas práticas e os contadores mediante sua atuação profissional.

Como restrições e limitações desta pesquisa, considera-se que não foram analisadas as pesquisas apresentadas em congressos da área contábil, assim como, dissertações e teses sobre o assunto. Para estudos futuros, sugere-se abordagens distintas daquelas encontradas nas pesquisas analisadas, como, por exemplo, a eficácia das operações de hedge divulgadas pelas empresas e o aumento da volatilidade dos resultados pelo uso dos derivativos.

\section{REFERÊNCIAS}

AMARAL, Carlos Antonio Lopes Vaz do. Derivativos: o que são e a evolução quanto ao aspecto contábil. Revista Contabilidade \& Finanças, São Paulo, n. 32, p. 71-80, 2003.

BARTH, Mary E.; LANDSMAN, Wayne R.; LANG, Mark H. International Accounting Standards and Accounting Quality. Journal of Accounting Research, v. 46, n. 3, 2008.

BURLÁ, Leonardo Andrade de Almeida; GONÇAVES, Edson Daniel Lopes. Gestão de risco e os impactos da instrução normativa CVM no 550 - análise empírica. Revista Contabilidade \& Finanças, São Paulo, v. 21, n. 53, p. 1-21, 2010.

CHATHAM, Michael D.; LARSON, Robert K.; VIETZE, Axel. Issues affecting the development of an international accounting standard on financial instruments. Advances in Accounting, incorporating Advances in International Accounting, v. 26, p. 97-107, 2010.

CHEW; Lillian. Gerenciando os riscos de derivativos: o uso e abuso da alavancagem. Tradução: Bruno Marsili. Rio de Janeiro: Qualitymark Ed., 1999. 
CPC, Comitê de Pronunciamentos Contábeis. OCPC 03 - Instrumentos Financeiros: Reconhecimento, Mensuração e Evidenciação (CPC 14 R1). 2009a. Disponível em: <www.cpc.org.br>. Acesso em: 20 abr 2014.

CPC, Comitê de Pronunciamentos Contábeis. CPC 38 - Instrumentos Financeiros: Reconhecimento e Mensuração. 2009b. Disponível em: <www.cpc.org.br>. Acesso em: 20 abr 2014.

CPC, Comitê de Pronunciamentos Contábeis. CPC 39 - Instrumentos Financeiros: Apresentação. 2009c. Disponível em: <www.cpc.org.br>. Acesso em: 20 abr 2014.

CPC, Comitê de Pronunciamentos Contábeis. CPC 40 (R1) - Instrumentos Financeiros:

Reconhecimento e Mensuração. 2012. Disponível em: <www.cpc.org.br>. Acesso em: 16 mai 2014.

CVM, COMISSÃO DE VALORES MOBILIÁRIOS. Instrução CVM no 235/95. 1995. Disponível em: <http://www.cvm.gov.br>. Acesso em: 01 abr 2014.

CVM, COMISSÃO DE VALORES MOBILIÁRIOS. Instrução CVM no 475/08. 2008. Disponível em: <http://www.cvm.gov.br>. Acesso em: 01 abr 2014.

DARÓS, Leandro Luís; BORBA, José Alonso. Evidenciação de instrumentos financeiros derivativos nas demonstrações contábeis: uma análise das empresas brasileiras. Revista Contabilidade \& Finanças, São Paulo, n. 35, p. 68-80, 2005.

DELOITTE. IASplus. Disponível em: <http://www.iasplus.com/en/standards>. Acesso em: 01 fev 2015.

COOPER, David J.; ROBSON, Keith. Accounting, professions and regulation: Locating the sites of professionalization. Accounting, Organizations and Society, v. 31, p. 415-444, 2006.

FASB, Financial Accounting Standards Board. International convergence of accounting standards - a brief history. Disponível em:

<http://www.fasb.org/jsp/FASB/Page/SectionPage\&cid=1176156304264>. Acesso em: 03 jul 2014.

FIPECAFI; ERNST \& YOUNG. Análises sobre o IFRS no Brasil - Edição 2013. Disponível em: $<$ http://www.ey.com/Publication/vwLUAssets/Análises_sobre_IFRS_no_Brasil/\$FILE/EY_Fip ecafi_2013_Web.pdf>. Acesso em: 16 maio 2014.

FIPECAFI; ERNST \& YOUNG. Estudo sobre as diferenças e similaridades entre as normas internacionais de contabilidade - IFRS e as normas e práticas contábeis brasileiras: Instrumentos Financeiros. 2008. Disponível em: <http://www.cvm.gov.br/>. Acesso em: 28 jun 2014.

GALDI, Fernando Caio; GUERRA, Luiz Fernando Grama. Determinantes para utilização de hedge accounting: uma escolha contábil. Revista de Educação e Pesquisa em Contabilidade, v. 3, n. 2, p. 23-44, 2009. 
IASB, International Accounting Standards Board. IAS 32 Financial Instruments: Presentation. 2003a. Disponível em: <http://www.ifrs.org>. Acesso em: 16 maio 2014.

IASB, International Accounting Standards Board. IAS 39 Financial Instruments: Recognition and Measurement. 2003b. Disponível em: < http://www.ifrs.org>. Acesso em: 16 maio 2014.

IFRS, International Financial Reporting Standards.Disponível em: < http://www.ifrs.org>. Acesso em: 16 maio 2014.

LOPES, Alexsandro Broedel; GALDI, Fernando Caio; LIMA, Iran Siqueira. Manual de contabilidade e tributação de instrumentos financeiros e derivativos. 2. ed. São Paulo: Atlas, 2011.

MARTINS, G. A.; THEÓPHILO, C. R. Metodologia da investigação científica para ciências sociais aplicadas. 2. ed. São Paulo: Atlas, 2009

NAKAMURA, Angela Mie. Contribuições ao estudo de procedimentos e evidenciação contábeis aplicáveis a operações com derivativos, voltados a instituições financeiras. São Paulo: Bolsa de Mercadorias \& Futuros, 2003.

SILVA, Tarcísio Pedro da; MACHADO, Débora Gomes; HEIN, Nelson. Análise exploratória da evidenciação da natureza e extensão dos instrumentos financeiros nas notas explicativas de empresas listadas na BM\&FBovespa. ConTexto, Porto Alegre, v. 13, n. 24, p. 37-49, 2013.

TAKAR, Téo. Bovespa bateu recorde de volume diário em setembro. Valor Econômico, 03/10/2012. Disponível em: <http://www.valor.com.br/financas/2854594/bovespa-bateurecorde-de-volume-diario-em-setembro\#ixzz36PNVdILQ>. Acesso em 03 jul 2014.

YOUNG, Joni J. Institutional thinking: the case of financial instruments. Accounting, Organizations and Society, v. 21, n. 5, p. 487-512, 1996. 